\title{
THE OPTIMAL TIMING OF BASELINE RADIOSTEREOMETRIC ANALYSIS OF UNCEMENTED PRESS FIT CUPS
}

\author{
O. Wolf, J. Milbrink, S. Larsson, P. Mattsson, H. Mallmin \\ Department of Orthopaedics, Uppsala University Hospital, Uppsala, Sweden
}

\begin{abstract}
Background and Aims: The baseline radiostereometric analysis (RSA) is usually performed during the first postoperative week. We investigated the micromotion of two uncemented press fit acetabular cups during the first week after total hip arthroplasty.

Material and Methods: All study patients had unilateral osteoarthritis of the hip and received an uncemented THA combination consisting of a CLS stem and either an Allofit or an Interop acetabular cup. The study group consisted of 24 patients who underwent RSA within 1 hour after skin closure, and at 1 and 7 days after surgery.

Results: The upper limit of the $95 \%$ confidence interval for micromotion was less than or close to the precision of the method for all studied directions during the first week after surgery. Mean values indicate proximal and medial translation of the uncemented cup at one week.

Conclusions: We found only minimal micromotion, barely above the precision limit, measured as medial and proximal translations of these uncemented cups. This indicates that the first postoperative RSA measurement following a primary THA with an uncemented press fit acetabular cup should be made as early as possible after the first postoperative day.
\end{abstract}

Key words: RSA; baseline examination; THA; uncemented cup; optimal timing; micromotion; weight bearing; muscle tonus

\section{INTRODUCTION}

Micromotion in hip arthroplasties can be determined with a high degree of precision by radiostereometric analysis (RSA) (1), and the method can predict future implant loosening of cemented femoral stems (2). The standard protocol regarding timing of the baseline examination is based on the assumption that no micromotion takes place during the first week after surgery. Examination one week after surgery has there-

Correspondence:

Olof Wolf, M.D.

Department of Orthopaedics

Uppsala University Hospital

SE - 75185 Uppsala, Sweden

Email: olof.wolf@akademiska.se fore been considered to represent a valid baseline when, over time, the total amount of micromotion from the time of surgery is assessed. For cemented implants, where stable implant fixation is achieved as soon as the cement has cured, this assumption is very reasonable. However, the validity of this baseline has not been tested for uncemented implants. In studies of uncemented implants, the baseline RSA examination has often been performed up to one week after surgery (3-5). It is therefore important to determine whether any micromotion of uncemented implants takes place during the interval from surgery up to the time at which the baseline protocol examinations are usually carried out, i.e., up to one week. So far, only one study of uncemented CLS stems (6) and one of femoral neck stability after internal fixation with screws (7) have been published in which RSA have been performed directly after surgery, while the pa- 
tient was still anesthetized and without or with reduced muscle tonus. The influence of muscle tonus in the hip joint is considerable even without weight bearing or during moderate exercise in bed $(8,9)$.

Early micromotions of the uncemented CLS press fit femoral stem (10) have previously been reported by our group (6). Since these micromotions were very small it was concluded that the one week examination would be appropriate as the baseline examination for uncemented stems (6). So far there have been no reports describing the micromotions of uncemented acetabular cups during the first week after surgery.

The primary aim of the present study was to determine whether any significant micromotion of uncemented acetabular cups occurred during the first week after surgery. Could the one week postoperative RSA be regarded as a reliable baseline RSA examination for an uncemented press fit acetabular cup?

\section{MATERIAL AND METHODS}

The patients included in the present study were part of a larger randomized controlled study of 45 patients undertaken with the aim of evaluating the impact of immediate unrestricted versus partial weight bearing on implant stability following uncemented total hip arthroplasty (THA). Inclusion criteria were unilateral osteoarthritis of the hip, age 25 to 65 years, and a body weight of less than $110 \mathrm{~kg}$. Exclusion criteria were use of steroids or other medication known to affect bone metabolism, malignancy, previous hip surgery, or BMI above 35 . Patients were randomized to either unrestricted postoperative weight bearing, including an intensive physiotherapy training program, or partial weight bearing for 3 months. Three of the 45 patients underwent surgery on the contralateral side within 5 years after the THA and were therefore excluded from the analysis. Because of problems at the start of the study in performing the one hour postoperative RSA, the number of patients was reduced. Thus, 29 of the remaining 42 patients underwent direct postoperative RSA while still under spinal anaesthesia and were therefore eligible for this study. Furthermore one more patient, who did not take part in the full study mentioned above, but who was followed with RSA, was included in this study. In total 30 patients were included. Enrolment took place between April 2000 and April 2003. Fifteen patients were randomized to unrestricted weight bearing and 15 to partial weight bearing. All patients gave informed consent.

All patients received the CLS hip stem (Centerpulse, Bern, Switzerland, acquired by Zimmer, Warsaw, IN). The original study included use of the uncemented Interop acetabular cup (Centerpulse, Bern, Switzerland, acquired by Zimmer Co, Warsaw, IN, USA) with a hemispherical porous shell with sealed screw holes, but manufacturing problems with oil contaminated shells (11) led to withdrawal of the product from the market. Thus, only 11 Interop acetabular cups were inserted. Although we had some clinical patients who had adverse effects from these oil-contaminated cups, no patients in the present study were affected. The study protocol was thus revised and the Allofit acetabular cup without screw holes (Centerpulse, Bern, Switzerland, acquired by Zimmer Co, Warsaw, IN, USA) was used for the remaining 19 patients. The Interop cup is a press fit cup with a modular hemispheric component with a roughened cancellized titanium back surface. The Allofit cup is also a press fit cup, but has a grit-blasted titanium surface. Both cups were combined with a polyethylene hooded insert. All CLS stems received a modular $28 \mathrm{~mm}$ cobalt-chrome head. Five experienced surgeons performed the operations in a standardized way in accordance with the manufacturer's manual, using an anterolateral approach.

\section{EXCLUSIONS}

All included 30 patients underwent all three planned RSA examinations, i.e., at 1 hour, 1 day and 7 days after surgery (see below). Three patients (1 Interop/ 2 Allofit) were excluded from the cup analysis because of inadequate visualization of markers. Three patients (2 Interop/ 1 Allofit) were excluded because of poor marker stability (i.e., the mean error (ME) was too high). Of the remaining 24 cups with reference examinations, two cups could not be evaluated at one day postoperatively (inadequate visualization of markers and poor scattering of markers, respectively) and one cup could not be evaluated at one week (poor scattering). In all, 21 cups could be analyzed at all three time points, and 24 cups at two of the three time points (22 at 1 day and 23 at 1 week).

\section{RADIOSTEREOMETRY}

The RSA procedure has been widely used for studying implant stability $(1,12)$. During surgery tantalum markers $(0.8$ $\mathrm{mm}$ in diameter) were inserted into the pelvis in a preplanned fashion. Cups were marked with 5-8 tantalum markers ( $1.0 \mathrm{~mm}$ in diameter) at the outer rim of the polyethylene hooded insert. In addition, the titanium shells were marked preoperatively with one tantalum marker on the central button at the bottom in a similar fashion in the Interop and the Allofit cups. Radiostereometric examinations were performed immediately after surgery with the patient still under spinal anaesthesia (" 1 hour examination"). Additional RSA examinations were carried out at 1 day and 1 week after surgery. A specially trained radiology research nurse performed all examinations. We used a uniplanar technique with the patient in the supine position, with the calibration cage 43 positioned under the examination table, and ceiling mounted $\mathrm{x}$-ray tubes positioned at an angle of $40^{\circ}$ with separate generators for true simultaneous exposure $(12,13)$. Initial radiographs were digitized by a scanner (UMAX, Umeå, Sweden). On account of modernization of the RSA X-ray laboratory, digital x-ray was performed in the last 6 patients. Loose markers, markers that are inadequately visualized by reason, for example, of hindering metal, or incorrect identification of markers, will all lead to measurement errors. The three-dimensional distribution of markers in each segment, i.e., the rigid body, is compared between examinations and measured as mean error (ME) of rigid body fitting $(1,14)$. Also, the distribution of markers in the investigated implant or bone, i.e. scattering, is important. The condition number $(\mathrm{CN})$ describes how well scattered the markers are in each segment (15). A low $\mathrm{CN}$ indicates good scattering of markers, and a high $\mathrm{CN}$ indicates markers close to each other or on a straight line. Examinations with a scatter corresponding to a $\mathrm{CN}$ of $<150$ and a stability corresponding to $\mathrm{ME}<0.3 \mathrm{~mm}$ were included for analysis. The precision of our RSA measurements in this study setup was based on 33 duplicate examinations of cups. On the basis of prior methodological reports on RSA precision, we calculated the $95 \%$ confidence limits to determine the lower limits of significant motions, i.e., the precision (Table 1) $(16,17)$. We used the unsigned values for micromotion in order to give a full picture of the possible translation and rotation, which would not be possible with signed values and mean values of micromotion. 
Micromotion of the cup was measured as rotations around three axes and as translations of the centre of the rigid body along these three axes. We used UmRSA Analysis 6.0 (RSA Biomedical, Umeå, Sweden) for measurements and calculations.

\section{STATISTICS}

Statistical analysis was performed with Statistica 8.0 (StatSoft, Inc, Tulsa, USA). Since we were interested in finding out whether micromotion occurred during the first week, regardless of direction, we used the unsigned (absolute) values to calculate the median and $95 \%$ confidence interval (CI) for the median. Confidence intervals for the median were based on order statistics (ranks) as described by Hahn and Meeker (18). These calculations were performed using SAS 9.1.3 software (SAS Institute Inc., Cary, North Carolina). We compared the $95 \%$ CI with the precision of the RSA procedure to determine whether the measured motion could be regarded as true motion. If the upper limit of the

TABLE 1

Precision for micromotion of the acetabular cups.

\begin{tabular}{ll}
\hline Type of motion & \\
Translation (mm) & 0.27 \\
$\quad$ Transverse axis (X) & 0.23 \\
$\quad$ Longitudinal axis (Y) & 0.34 \\
Sagittal axis (Z) & \\
Rotation (degrees) & 0.87 \\
$\quad$ Transverse axis (X) & 1.17 \\
$\quad$ Longitudinal axis (Y) & 0.54 \\
Sagittal axis (Z) &
\end{tabular}

95\% prediction interval about zero for significant motions based on 33 duplicate examinations
95\% CI was lower than the precision limit, no significant micromotion has occurred and the one week RSA examination can be regarded as a reliable baseline (Table 2). Although the study was not designed for this purpose, we also carried out analyses to find out whether any observed micromotion was dependent on the sort of cup used or the kind of postoperative weight bearing regime. For these analyses we used the Mann-Whitney U-test for the unsigned values. P-values of less than .05 were considered significant.

The local ethics committee approved the investigation prior to the start of the study (Ups 99242).

\section{RESULTS}

After exclusions, the study group consisted of 24 patients (14 men) with a mean age of 54.1 (SD 9) years, a mean weight of 79.9 (SD 12) kg and a mean height of 172.7 (SD 10) cm. There was no difference in age, weight, or height between subgroups depending on weight bearing or cup (Subgroups, see Table 3).

\section{CUP MIGRATION (TABLE 2)}

The upper limit of the $95 \%$ confidence interval for micromotion was lower than the precision at one day and one week except for translation along the $X$-axis (medial-lateral) and Y-axis (proximal-distal) at 1 week and rotation around the $Z$-axis (inclination) at 1 day. The absolute median (unsigned) values for micromotion along and around the three axes were all below precision. Also, the mean values (signed) for translation along the $\mathrm{X}$-axis and $\mathrm{Y}$-axis were both just above the precision limit. Individual translations and rota-

TABLE 2

Micromotion of the uncemented acetabular cups at 1 day $(n=22)$ and 1 week $(n=23)$ in comparison with that at the baseline RSA examination one hour postoperatively. An upper limit of the $95 \%$ confidence interval below the precision limit indicates no significant micromotion.

\begin{tabular}{|c|c|c|c|c|}
\hline & Mean (range) & Median (abs.) & $95 \%$ CI (abs.) & Precision \\
\hline $\begin{array}{l}\text { Translation }(\mathrm{mm}) \\
\text { Medial (+)/lateral (-) } \\
1 \text { day } \\
1 \text { week }\end{array}$ & $\begin{array}{ll}0.21 & (-0.15-1.35) \\
0.31 & (-0.59-1.36)\end{array}$ & $\begin{array}{l}0.086 \\
0.213\end{array}$ & $\begin{array}{l}0.035-0.211 \\
0.058-0.576\end{array}$ & 0.27 \\
\hline $\begin{array}{l}\text { Proximal (+)/distal }(-) \\
1 \text { day } \\
1 \text { week }\end{array}$ & $\begin{array}{ll}0.14 & (-0.03-0.89) \\
0.27 & (-0.13-1.10)\end{array}$ & $\begin{array}{l}0.034 \\
0.115\end{array}$ & $\begin{array}{l}0.026-0.134 \\
0.064-0.371\end{array}$ & 0.23 \\
\hline $\begin{array}{l}\text { Anterior }(+) / \text { posterior }(-) \\
1 \text { day } \\
1 \text { week }\end{array}$ & $\begin{array}{ll}-0.07 & (-0.51-0.22) \\
-0.09 & (-0.51-0.40)\end{array}$ & $\begin{array}{l}0.078 \\
0.128\end{array}$ & $\begin{array}{l}0.042-0.170 \\
0.053-0.290\end{array}$ & 0.34 \\
\hline $\begin{array}{l}\text { Rotation (degrees) } \\
\text { Anterior (+)/posterior (-) tilt } \\
1 \text { day } \\
1 \text { week }\end{array}$ & $\begin{array}{ll}-0.14 & (-1.76-0.66) \\
-0.06 & (-1.45-1.17)\end{array}$ & $\begin{array}{l}0.315 \\
0.390\end{array}$ & $\begin{array}{l}0.180-0.520 \\
0.180-0.800\end{array}$ & 0.87 \\
\hline $\begin{array}{l}\text { Anteversion }(+) \\
\text { retroversion }(-) \\
1 \text { day } \\
1 \text { week }\end{array}$ & $\begin{aligned} 0.13 & (-1.53-1.86) \\
-0.02 & (-2.05-1.88)\end{aligned}$ & $\begin{array}{l}0.240 \\
0.240\end{array}$ & $\begin{array}{l}0.210-0.540 \\
0.120-0.430\end{array}$ & 1.17 \\
\hline $\begin{array}{l}\text { Decreased }(+) / \text { increased }(-) \\
\text { Inclination } \\
1 \text { day } \\
1 \text { week }\end{array}$ & $\begin{array}{ll}0.35 & (-0.17-1.63) \\
0.21 & (-1.88-2.33)\end{array}$ & $\begin{array}{l}0.140 \\
0.340\end{array}$ & $\begin{array}{l}0.070-0.550 \\
0.150-0.520\end{array}$ & 0.54 \\
\hline
\end{tabular}

Mean values and ranges are signed values. Median of absolute (abs.) values (unsigned) and 95\% confidence intervals (CI) of absolute median are given. The precision of the RSA method in this study setup is based on 33 duplicate examinations. 
TABLE 3

Patient characteristics by sex, type of cup and degree of weight bearing.

\begin{tabular}{lrlll}
\hline & $\mathrm{n}$ & Age, years & Weight, kg & Height, cm \\
\hline Men & 14 & $57.4(3.8)$ & $85.8(8.4)$ & $179.1(5.7)$ \\
Women & 10 & $49.4(12.4)$ & $71.6(10.5)$ & $163.7(5.5)$ \\
Interop & 8 & $52.7(8.1)$ & $82.5(7.8)$ & $173.4(9.2)$ \\
Allofit & 16 & $54.8(9.9)$ & $81.0(13.1)$ & $172.3(10.0)$ \\
Full WB & 12 & $53.1(12.5)$ & $78.7(11.0)$ & $174.1(10.1)$ \\
Partial WB & 12 & $55.0(4.4)$ & $171.2(9.1)$ \\
\hline
\end{tabular}

Mean values (SD). WB= weight bearing. No differences between cup or WB groups.
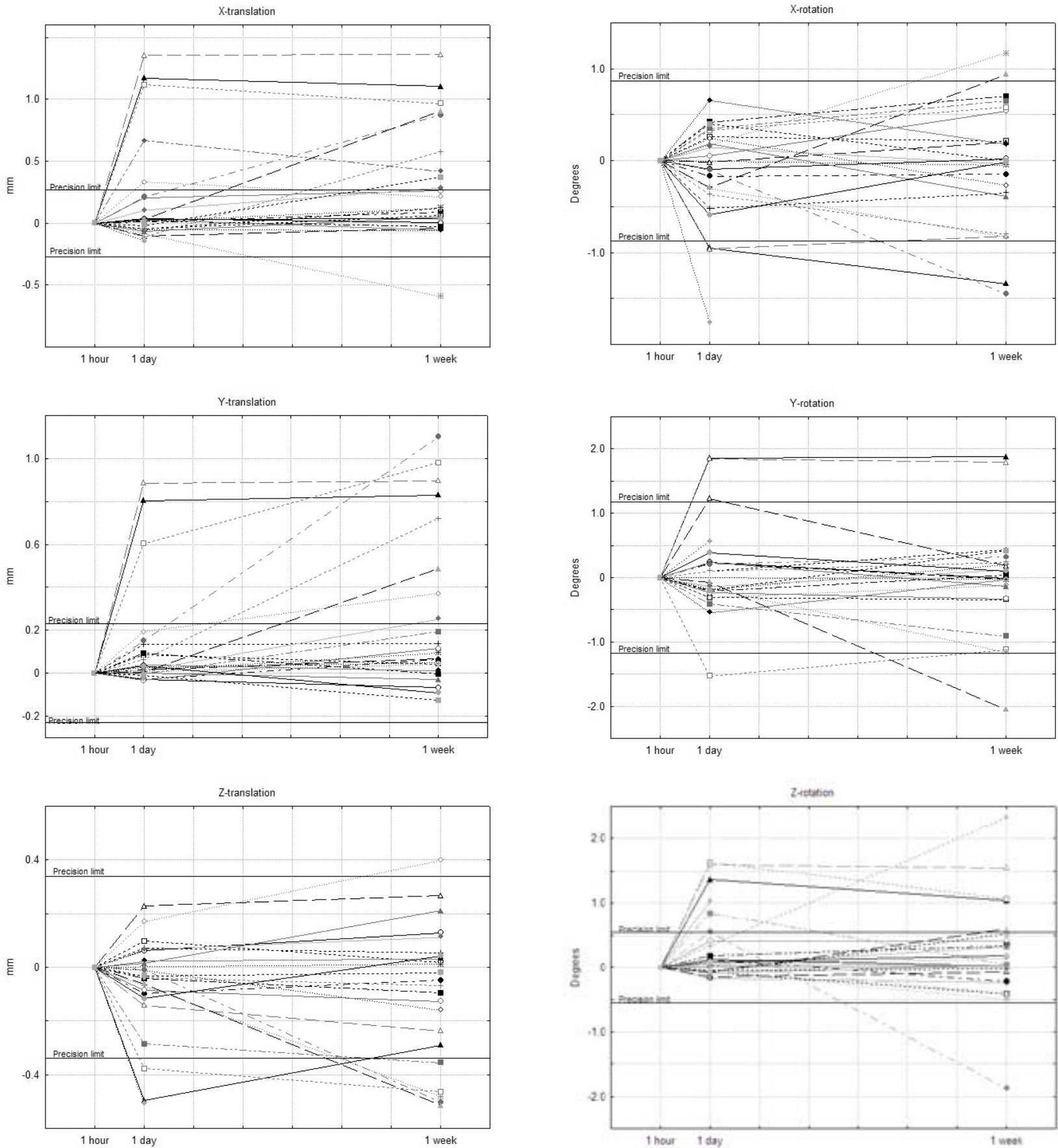

Fig 1. Individual translations and rotations of 24 cups along the 3 axes. Precision limits are indicated. 
tions along the $\mathrm{X}$-axis, $\mathrm{Y}$-axis, and $\mathrm{Z}$-axis are presented in Fig. 1.

The mean ME was 0.095 (range 0.011-0.289) and the mean $\mathrm{CN}$ was 59 (21-148). We found no difference in micromotion (mean values) between unrestricted weight bearing and partial weight bearing (data not shown). At one week there was a difference between cups in mean medial translation (Interop $0.18 \mathrm{~mm}$, Allofit $0.48 \mathrm{~mm}, \mathrm{p}<0.05)$.

\section{DISCUSSION}

The mean values for both cups together indicate proximal and medial micromotion, i.e., in the direction of loading. However, the values only barely exceeded the precision limits. The upper limit for the 95\% CI for micromotions along the X- and Y-axes after one week and for rotation around the Z-axis after one day exceeded the precision.

There was no difference in implant micromotion between patients who were randomized to full weight bearing and intensive physiotherapy and those who were allocated to restricted weight bearing and limited physiotherapy after surgery. Comparisons between the two different cups revealed differences in one direction, i.e., medial translation.

As uncemented implants rely on different fixation concepts, the results from the current study might be restricted to implants with similar press fit designs. The present results cannot therefore be generalized to represent all uncemented cups.

In several studies on acetabular cup stability after primary THA the baseline RSA measurements have been performed 3-7 days postoperatively (3-5). Although RSA is a very sensitive method that is able to detect small changes in position, i.e., micromotion, there have been very few reports of early changes in position from RSA studies. The rationale for the timing of the index RSA measurement is in general unclear. Ornstein et al (19) found that most of the micromotion in their study on hip revisions with morcelized allograft and cement occurred during the first two weeks after surgery, with the index RSA examination one day postoperatively, but prior to mobilization. The limit for significant micromotion was again the precision limit in their study, with cup precisions of similar magnitude to those in our study. They concluded that it was important to specify the postoperative regime and at least for revision surgery, that the index measurement should be made on the $1^{\text {st }}$ or $2^{\text {nd }}$ day postoperatively. However, the bone stock in a revised acetabulum is more likely to be inferior compared to that in a primary replacement, suggesting differences in biomechanical properties. In a previous study (6) no micromotion of the uncemented CLS stem in primary THA was found during the first week after surgery, whether the patients were instructed to use full or partial weight bearing. In the present study we have focused on micromotion during the very early postoperative period with the aim of determining whether any significant micromotion of uncemented acetabular cups occurs during the first week after THA. In an attempt to investigate whether muscle tonus could influence the optimal time for baseline RSA, this baseline examination was performed with the patients still under the effects of the spinal anaesthesia. The initial stability of the uncemented implants used in this study seemed to withstand the forces of muscle tonus well. The different postoperative regimes, full and partial weight bearing, may, on account of self-limited pain restrictions, perhaps not differ greatly during the first days following surgery.

This study has some limitations. The study design with two different postoperative regimes could influence micromotion. Although patients allocated to the full weight bearing group put more weight on the leg after one week (20), we found no differences in micromotion between the two groups during the first week either of the cup, or of the stem (6). In order to increase the statistical power we therefore combined the patients with the two postoperative regimes for the primary analysis. The original study protocol was violated on account of factors out of our control. We were forced to stop using the Interop cups and switched to the Allofit cup; i.e., the protocol was changed to include two rather than only one type of uncemented acetabular cup. Thus, the already small sample size was reduced. When the cups were analyzed separately, despite the low number of patients, differences between them regarding excess of the precision limit for medial and proximal translation were detected at the one week examination. Still, since the cups were of the same material and of similar type, i.e., press fit, although with different kinds of surfaces but from the same manufacturer, we also combined the two different cups for the primary analysis. The mean values were small overall and this might be true for more than one type of uncemented press-fit cup. However, these conclusions could be criticized on the ground of the limited sample size.

This study also has certain merits. It is the first study that has focused on the very early motions of uncemented acetabular cups in primary THA evaluated with the sensitive RSA method. The first RSA measurement, performed within one hour after skin closure and with the patient still under spinal anaesthesia, was a demanding procedure. The patients had to be taken directly from the operating room to an X-ray department equipped for RSA and with skilled staff, situated near the operation ward.

To conclude, we found only minimal micromotion, barely above the precision limit, measured as medial and proximal translations of these uncemented cups. On the basis of these results, we conclude that the first postoperative RSA measurement in a primary THA with an uncemented press fit acetabular cup should be made as early as possible after the first postoperative day.

\section{ACKNOWLEDGEMENTS}

This study could not have been performed without the strenuous work of our late colleague Dr. Håkan Ström. 
We are grateful to Monika Gelotte, RN, for skilful RSA measurements.

We wish to thank Lisa Wernroth, at the Uppsala Clinical Research Center, for valuable statistical assistance.

Zimmer Inc. sponsored the study financially. They had no influence on the design of the study, collection of data, or writing of the manuscript.

\section{REFERENCES}

1. Selvik G: Roentgen stereophotogrammetry. A method for the study of the kinematics of the skeletal system. Acta Orthop Scand Suppl 1989;232:1-51

2. Kärrholm J, Malchau H, Snorrason $F$ et al: Micromotion of femoral stems in total hip arthroplasty. A randomized study of cemented, hydroxyapatite-coated, and porous-coated stems with roentgen stereophotogrammetric analysis. J Bone Joint Surg Am 1994;76:1692-1705

3. Thien TM, Ahnfelt L, Eriksson M et al: Immediate weight bearing after uncemented total hip arthroplasty with an anteverted stem: a prospective randomized comparison using radiostereometry. Acta Orthop 2007;78:730-738

4. Rohrl SM, Nivbrant B, Strom H et al: Effect of augmented cup fixation on stability, wear, and osteolysis: a 5-year follow-up of total hip arthroplasty with RSA. J Arthroplasty, 2004;19: 962-971

5. Palm L, Olofsson J, Astrom SE et al: No difference in migration or wear between cemented low-profile cups and standard cups: a randomized radiostereographic study of 53 patients over 3 years. Acta Orthop 2007;78:479-484

6. Ström H, Nilsson O, Milbrink J et al: Early migration pattern of the uncemented CLS stem in total hip arthroplasties. Clin Orthop Relat Res 2007;454:127-132

7. Ragnarsson JI, Hansson LI,Karrholm J: Stability of femoral neck fractures. A postoperative roentgen stereophotogrammetric analysis. Acta Orthop Scand 1989;60:283-287

8. Rydell N: Biomechanics of the hip-joint. Clin Orthop Relat Res 1973:6-15
9. Bergmann G, Rohlmann A,Graichen F: [In vivo measurement of hip joint stress. 1. Physical therapy]. Z Orthop Ihre Grenzgeb 1989;127:672-679

10. Spotorno L, Romagnoli S, Ivaldo $\mathrm{N}$ et al: The CLS system. Theoretical concept and results. Acta Orthop Belg 1993;59 Suppl 1:144-148

11. Blumenfeld TJ,Bargar WL: Early aseptic loosening of a modern acetabular component secondary to a change in manufacturing. J Arthroplasty 2006;21:689-695

12. Kärrholm J, Herberts $P$, Hultmark $P$ et al: Radiostereometry of hip prostheses. Review of methodology and clinical results. Clin Orthop Relat Res 1997:94-110

13. Valstar ER, Gill R, Ryd L et al: Guidelines for standardization of radiostereometry (RSA) of implants. Acta Orthop 2005;76: 563-572

14. Karrholm J: Roentgen stereophotogrammetry. Review of orthopedic applications. Acta Orthop Scand 1989;60:491-503

15. Onsten I, Carlsson AS, Sanzen L et al: Migration and wear of a hydroxyapatite-coated hip prosthesis. A controlled roentgen stereophotogrammetric study. J Bone Joint Surg Br 1996;78: 85-91

16. Derbyshire B, Prescott RJ,Porter ML: Notes on the use and interpretation of radiostereometric analysis. Acta Orthop 2009; $80: 124-130$

17. Ranstam J, Ryd L,Onsten I: Accurate accuracy assessment: review of basic principles. Acta Orthop Scand 2000;71:106108

18. Hahn GJ,Meeker WQ: Statistical intervals: a guide for practitioners. 1991, New York: John Wiley \& Sons Inc

19. Ornstein E, Franzen H, Johnsson R et al: Radiostereometric analysis in hip revision surgery - optimal time for index examination: 6 patients revised with impacted allografts and cement followed weekly for 6 weeks. Acta Orthop Scand 2000; 71:360-364

20. Ström H, Huss K,Larsson S: Unrestricted weight bearing and intensive physiotherapy after uncemented total hip arthroplasty. Scand J Surg 2006;95:55-60

Received: January 29, 2010

Accepted: April 26, 2010 